\title{
Diagnostic value of contrast-enhanced ultrasound in papillary thyroid microcarcinoma
}

\author{
HONG YAN CHEN ${ }^{1}$, WEI YAN LIU ${ }^{2}$, HUI ZHU ${ }^{1}$, DAO WEN JIANG ${ }^{2}$, DONG HUA WANG ${ }^{1}$, \\ YONGQI CHEN ${ }^{3}$, WEIHUA LI ${ }^{1}$ and GAOFENG PAN ${ }^{2}$
}

Departments of ${ }^{1}$ Ultrasound in Medicine, ${ }^{2}$ Surgery and ${ }^{3}$ Pathology, Minhang Central Hospital, Shanghai 201199, P.R. China

Received March 21, 2015; Accepted November 25, 2015

DOI: 10.3892/etm.2016.3094

\begin{abstract}
The aim of the present study was to explore the value and characteristics of contrast-enhanced ultrasound (CEUS) in the diagnosis of papillary thyroid microcarcinoma (PTMC). By analyzing CEUS information of 130 nodules obtained from 106 patients with PTMC, who had been diagnosed by surgery and pathological analysis, CEUS characteristics of PTMC nodules were concluded. Based on the results, the PTMC nodules were divided into three groups as follows: 32 nodules $(24.62 \%)$ were found to be enhanced earlier than the surrounding normal thyroid tissue, 95 nodules $(73.08 \%)$ were enhanced at the same time as the normal thyroid tissue and 3 nodules $(2.30 \%)$ were enhanced later than the normal thyroid tissue. The results also demonstrated that the peak enhancement intensity of the 130 nodules was lower compared with the irregular intensity of the normal parenchyma in corresponding thyroids, and that PTMC enhancement washed out faster than in normal thyroid parenchyma. In conclusion, the PTMC characteristics that CEUS can detect may improve the diagnostic accuracy and provide valuable information for the treatment of the disease.
\end{abstract}

\section{Introduction}

The incidence of thyroid cancer has gradually increased in recent years, and it is currently the most common endocrine tumor (1). Thyroid microcarcinoma is defined as a thyroid tumor $\leq 10 \mathrm{~mm}$ in diameter, and $65-99 \%$ of these tumors are papillary thyroid microcarcinomas (PTMCs) (2). In recent years, the incidence of papillary thyroid carcinoma has increased significantly, specifically for PTMC (by $24 \%$ in patients aged $>45$ years) (3). The diagnosis of PTMC relies on imaging techniques, since the lesions are difficult to assess and easily misdiagnosed

Correspondence to: Dr Hong Yan Chen, Department of Ultrasound in Medicine, Minhang Central Hospital, 170 Xin Song Road, Shanghai 201199, P.R. China

E-mail: chyminhang@163.com

Key words: thyroid papillary microcarcinoma, contrast-enhanced ultrasonography, diagnosis due to their small size (4). In particular, ultrasound examination is preferred for small thyroid lesions, and quantitative contrast-enhanced ultrasound (CEUS) can clearly define the microvascularity of small carcinoma nodules (5). The diagnosis rate using palpation is low and the diagnostic specificity of computed tomography/magnetic resonance imaging (CT/MRI) is poor when PTMC nodules are $<10 \mathrm{~mm}$ in diameter (2). Therefore, the aim of the present study was to investigate the value of CEUS in the diagnosis of PTMC.

\section{Materials and methods}

Patient samples. Between July 2013 and April 2014, 106 patients (14 male, 92 female) with a total of 130 thyroid nodules treated at the Minhang Central Hospital (Shanghai, China) were included in this study. All patients underwent CEUS prior to surgery and pathological diagnosis. The mean age of the patients was 41 years (age range, 23-66 years), and the mean diameter of the thyroid nodules was $6.1 \mathrm{~mm}$ (range, $1.9-10 \mathrm{~mm}$; 54 nodules measured 2-5 $\mathrm{mm}$ and 76 nodules measured 5-10 $\mathrm{mm}$ in diameter). In total, 12 patients had two nodules and 5 patients had multiple nodules. Following two-dimensional and color Doppler ultrasound examination, a nodule diameter of $\geq 10 \mathrm{~mm}$ with calcification, or a longitudinal/transverse length $>1$ was characteristic of malignant nodules. All PTMC nodules were confirmed by surgery and pathological analysis. Following the surgical procedure the tissue samples were fixed with $10 \%$ neutral formaldehyde (Shanghai Xi Hua Trade Co., Ltd., Shanghai, China) prior to being embedded with paraffin (Long Tu Was Industry Co., Ltd., Shanghai, China), sectioned using a Leica-RM2235 microtome (Leica Microsystems GmbH, Wetzlar, Germany) and stained with hematoxylin and eosin (Hongqiao Shanghai Lexiang Medical Reagent Co., Ltd., Shanghai, China). The sections were then observed and images were captured using a microscope (Olympus-BX51; Olympus Corporation, Tokyo, Japan). Pathological diagnosis was conducted based on the presence of the following: Papillary tumor cell growth, ground-glass opacity nuclei and nuclear grooves.

Imaging. High resolution B-mode and color Doppler ultrasound imaging of the thyroid gland was performed by an experienced examiner using a linear ultrasound transducer (5-12 MHz; Aplio XG SSA 790A; Toshiba Corp., Tokyo, Japan). Three ultrasound nodule characteristics were associated with a risk 
Table I. Sonographic characteristics of papillary thyroid microcarcinoma.

\begin{tabular}{lcccr}
\hline & \multicolumn{2}{c}{ Nodule diameter } & & \\
\cline { 2 - 3 } Parameter & $2-5 \mathrm{~mm}$ & $5-10 \mathrm{~mm}$ & $\chi^{2}$-test & P-value \\
\hline Echo & & & & \\
$\quad$ Homogeneous hypoechoic & 54 & 23 & 63.578 & 0.001 \\
$\quad$ Inhomogeneous hypoechoic & 0 & 53 & 63.578 & 0.001 \\
Round or not & 5 & & & 0.001 \\
$\quad$ Yes & 49 & 64 & 71.208 & 0.001 \\
No & & 12 & 71.208 & 0.001 \\
Longitude/transverse & 49 & 12 & 71.208 & 0.001 \\
$>1.5$ & 5 & 64 & 71.208 & 0.001 \\
$<1.5$ & 2 & & & 0.001 \\
Microcalcifacation & 52 & 37 & 30.415 & \\
Yes & & 39 & & \\
No & & & & \\
\hline
\end{tabular}

of thyroid cancer: Micro calcifications, size $>2 \mathrm{~cm}$ and solid composition (6). Following B-mode and Doppler evaluation, CEUS examination was performed. Instrument parameters were kept consistent and were as follows: 0.19 mechanical index, $3.5 \mathrm{~cm}$ depth and overall gain between 72 and $80 \%$. Prior to CEUS examination, patients were administered an intravenous bolus of $2.4 \mathrm{ml}$ SonoVue (Bracco S.p.A., Milan, Italy) followed by a bolus injection of $5 \mathrm{ml} 0.9 \% \mathrm{NaCl}$. Digital cine loops were recorded and stored for 3 min starting immediately after SonoVue administration, which allowed a retrospective independent evaluation to be conducted by two physicians with $>10$ years of experience in ultrasonic diagnosis. The parameters recorded and reviewed included the arrival time, enhancement intensity, internal enhancement, edge enhancement and wash-out time of SonoVue in PTMC nodules.

Arrival time. The perfusion time between the SonoVue injection and its appearance in the nodules was recorded as the arrival time. The patients were divided into three groups based on the relative arrival time: Earlier, same or later arrival time compared with that surrounding the normal thyroid parenchyma.

Enhancement intensity. The peak enhancement intensity of the PTMC nodules and the surrounding normal thyroid parenchyma were analyzed and compared. Peak enhancement intensity refers to the patterns and features of PTMC at peak time. In total, there were three PTMC nodule enhancement groups as follows: Hypo-enhancement, iso-enhancement and hyper-enhancement. In hypo-enhancement the peak value and the area under the curve of the nodules were lower than the normal surroundings tissues. In iso-enhancement the peak value and the area under the curve of the nodules were the same as the normal surroundings tissues. In hyper-enhancement the peak value and the area under the curve of the nodules were higher than the normal surroundings tissues

Internal enhancement. The internal enhancement of PTMC nodules was determined in relation to the homogeneity of internal enhancement when peak enhancement was reached. The ultrasound images were divided into three groups as follows: Homogeneous enhancement (the nodule exhibited a homogeneous uptake of the contrast medium), inhomogeneous enhancement (the nodule exhibited inhomogeneous uptake of the contrast medium) and local non-enhancement (the nodule did not take up the contrast medium).

Edge enhancement. According to the region intensity and uniformity of the PTMC nodule edge enhancement, the ultrasound images were divided into three groups as follows: Edge regular enhancement, irregular enhancement and non-enhancement. If the nodule and the surrounding normal tissue enhancement were similar, with no clear boundaries, no edge enhancement was present.

Wash-out time. The fading time of the SonoVue agent in the PTMC nodules was compared with the corresponding fading time of the agent in normal thyroid parenchyma. The ultrasound images obtained were divided into three groups as follows: Earlier, same and later wash-out time compared with that in the normal thyroid parenchyma.

Statistical analysis. Statistical analysis was performed using SPSS version 13.0 statistical software (SPSS, Inc., Chicago, IL, USA). Comparisons of diagnostic accuracy were performed using the $\chi^{2}$ test. $\mathrm{P}<0.05$ was considered to indicate a statistically significant difference.

\section{Results}

Imaging. Conventional B-mode ultrasound displayed hypoechogenicity in 77 PTMC nodules, 53 of which were inhomogeneous. A total of 69 nodules were round 5 nodules ranging between 2 and $5 \mathrm{~mm}$; 64 nodules ranging between 5 and $10 \mathrm{~mm}$ ), the aspect ratio (longitudinal diameter/transverse diameter) of 61 nodules was $>1.5$ (49 nodules between 2 and $5 \mathrm{~mm} ; 12$ nodules ranging between 5 and $1 \mathrm{~mm}$ ) and 39 nodules 
Table II. Contrast-enhanced ultrasonic arrival time, time-to-peak and wash-out time of papillary thyroid microcarcinoma.

\begin{tabular}{lccr}
\hline Parameters & Papillary thyroid carcinoma & Normal thyroid tissue & t-value \\
\hline Arrival time & $13.3 \pm 3.2$ & $13.7 \pm 3.2$ & 5.6928 \\
Time-to-peak & $20.8 \pm 4.6$ & $21.7 \pm 4.6$ & 10.8288 \\
Wash-out time & $26.8 \pm 5.4$ & $29.1 \pm 5.4$ & 17.8237
\end{tabular}

Data are presented as the mean \pm standard deviation.

Table III. Contrast-enhanced ultrasonic enhancement and fading patterns of papillary thyroid microcarcinoma.

\begin{tabular}{lcc}
\hline & \multicolumn{2}{c}{ Diameter } \\
\cline { 2 - 3 } Parameter & $2-5 \mathrm{~mm}(\mathrm{n}=54)$ & $5-10 \mathrm{~mm}(\mathrm{n}=76)$ \\
\hline Enhancement patterns & 12 & 20 \\
Early & 41 & 54 \\
Simultaneous & 1 & 2 \\
Late & & \\
Enhancement intensities & 52 & 73 \\
Hypo-enhancement & 2 & 2 \\
Iso-enhancement & 0 & 1 \\
Hyper-enhancement & & 0 \\
Internal enhancement & 2 & 76 \\
Homogenous & 52 & 0 \\
Inhomogenous & 0 & 0 \\
Non-enhanced & & 0 \\
Fading patterns & 54 & \\
Early wash-out time & 0 & \\
Synchronized & & \\
Slow wash-out time & & \\
\hline
\end{tabular}

showed microcalcifications ( 2 nodules ranging between 2 and $5 \mathrm{~mm}$; 37 nodules ranging between 5 and $10 \mathrm{~mm}$ ). Color Doppler demonstrated that the blood flow was undetectable in 31 nodules (Fig. 1A) and limited in 82 nodules (Fig. 1B), while a rich blood flow signal was observed in 17 nodules (Fig. 1C). A narrow strip-like or short-rod type blood flow was observed in 76 PTMC nodules (Fig. 1D). Table I presents the sonographic characteristics of PTMC.

Arrival time, time-to-peak and wash-out time. The arrival time, time-to-peak (time taken for the contrast agent to reach its highest intensity in the nodules) and wash-out time of PTMC nodules ranged between 10.1-16.5 sec (mean time, $13.3 \pm 3.2 \mathrm{sec}$ ), 16.2-25.4 sec (mean time, 20.8 $\pm 4.6 \mathrm{sec}$ ) and 21.4-32.2 sec (mean time, $26.8 \pm 5.4 \mathrm{sec}$ ), respectively (Table II). The arrival time, time-to-peak and wash-out time of normal thyroid tissue ranged between 10.5-16.9 sec (mean time, $13.7 \pm 3.2 \mathrm{sec}$ ), 17.1-26.3 sec (mean time, 21.7 $\pm 4.6 \mathrm{sec}$ ) and 23.7-34.5 sec (mean time, 29.1 $+5.4 \mathrm{sec}$ ), respectively (Table II). The difference in the arrival, time-to-peak and wash-out times between PTMC nodules and normal thyroid tissues was
A

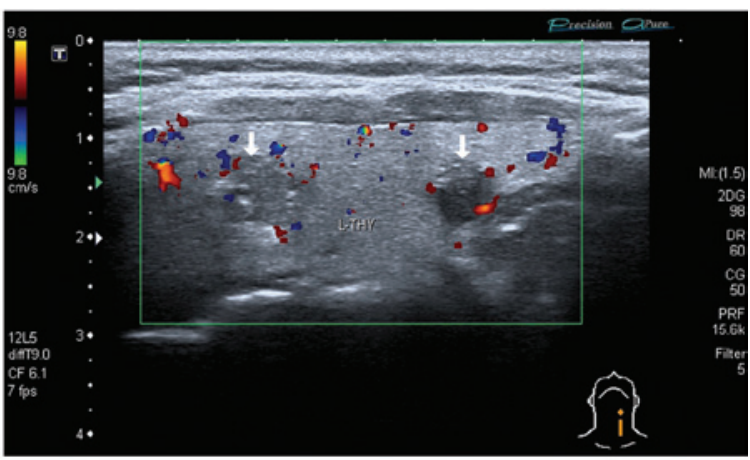

B

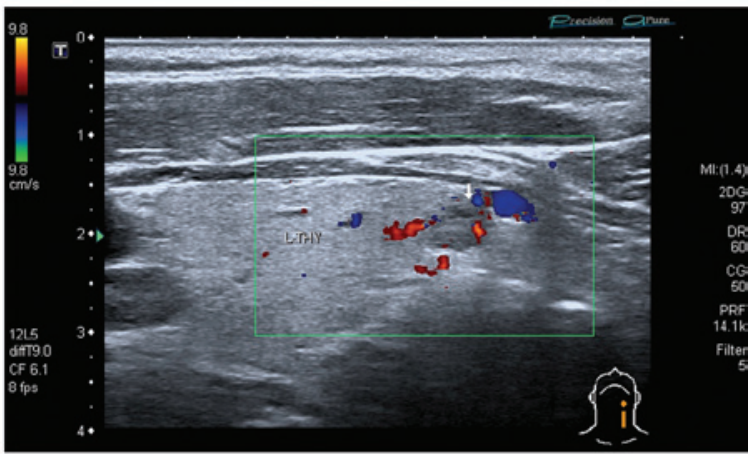

C

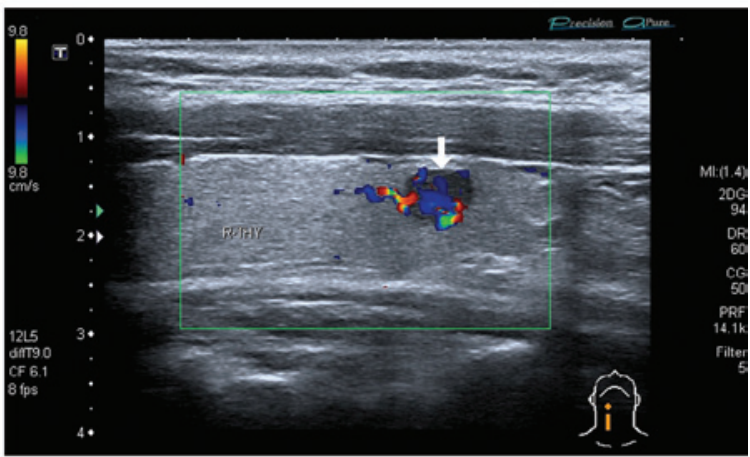

D

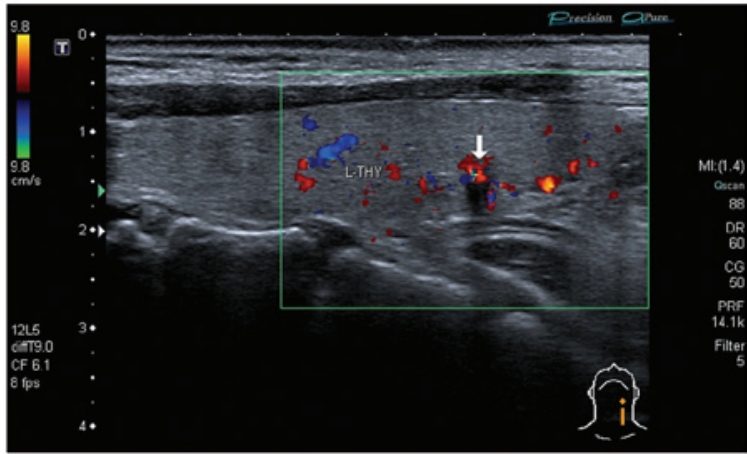

Figure 1. Papillary thyroid microcarcinoma has four types of Color Doppler imaging, indicating: (A) No evident blood flow signals in the nodule; (B) limited blood flow signal in the nodule; (C) rich blood flow signal in the nodule; and (D) a narrow strip-like or short rod-like blood flow penetrating into the nodule. White arrows indicate nodule location and green lines CDFI frames. 

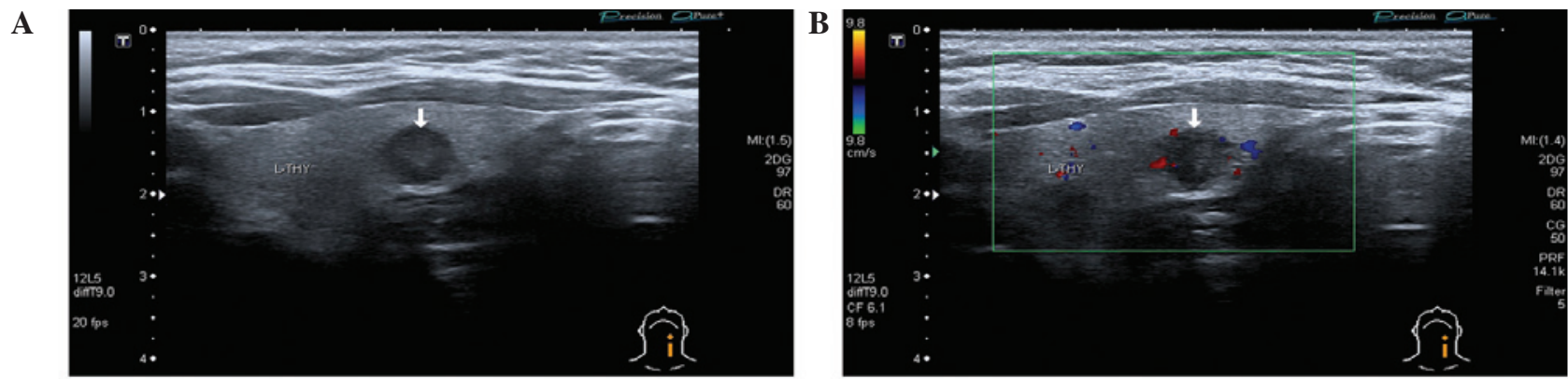

C

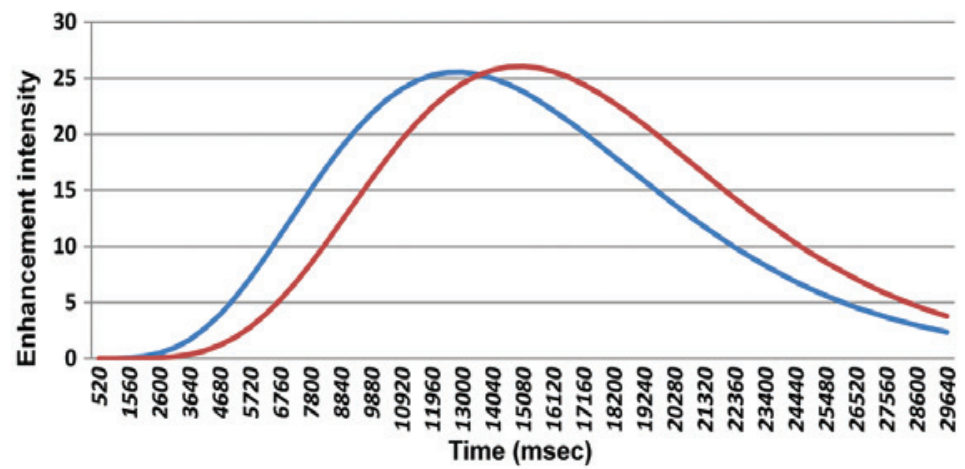

D

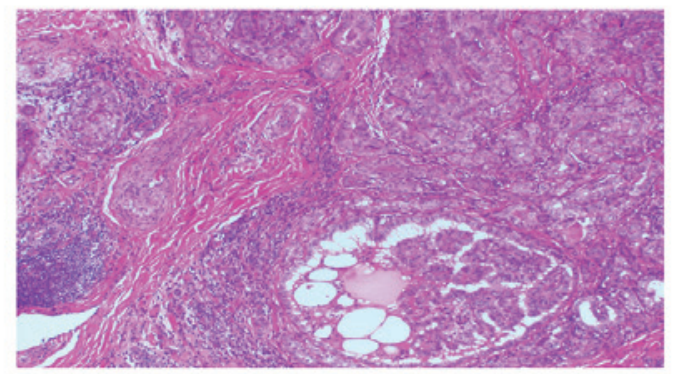

Figure 2. Sonograms of papillary thyroid microcarcinoma with (A) an ill-defined, irregular, round hypoechoic nodule (B-mode ultrasound). (B) The hypoechoic nodule exhibits peripheral punctate or short-rod like blood flow signal (color Doppler ultrasound). (C) The enhancement mode of the hypoechoic nodule arrived earlier and washed out faster than the surrounding normal thyroid tissue. The blue curve represents the thyroid nodule and the red curve the surrounding normal thyroid tissue. Compared with the surrounding normal thyroid tissue, the slope of the ascending and descending branches were higher for the thyroid nodule. (D) Hematoxylin and eosin staining demonstrating the congestion of thick vascular inside a papillary cancer nodule. White arrows indicate the lesion and green lines the CDFI frame.

statistically significant $(\mathrm{P}=0.0001)$. Therefore, arrival time, time to peak and wash-out time in the PTMC nodules were earlier compared with the surrounding normal tissues.

Contrast-enhancement patterns. Based on the enhancement and wash-out times, the contrast-enhanced patterns of 130 PTMC nodules were divided into three groups as follows: 32 nodules $(24.62 \%)$ were enhanced earlier than normal thyroid tissues (Fig. 2), 95 nodules (73.08\%) were enhanced at the same time as normal thyroid tissues (Fig. 3) and 3 nodules $(2.30 \%)$ were enhanced later than normal thyroid tissues (Fig. 4). All PTMC nodules washed out earlier than normal thyroid tissues.

Imaging features. The imaging features of 130 PTMC nodules from 106 patients were as follows: 125 nodules displayed low internal enhancement with fuzzy edge enhancement or irregular edge enhancement, which washed out faster than in normal thyroid tissues. A further 4 nodules displayed iso-enhancement, and 1 hyper-enhancement. The perfusion pattern mainly displayed dot or short rod inhomogeneity enhancement. This may be attributed to the small size of the nodules (diameter, $\leq 5 \mathrm{~mm}$ ), which failed to form the tumor vascular beds and arteriovenous fistulas that are required to provide sufficient blood supply to PTMCs. The CEUS enhancement and fading patterns of PTMCs are presented in Table III.

Ultrasound comparisons. Table IV presents the diagnosing values of B-mode ultrasound, color Doppler and CEUS. No statistically significant differences were identified between 2D ultrasound alone or combined with color Doppler in their ability to differentiate between $2-5 \mathrm{~mm}\left(\chi^{2}=1.069\right.$, $\mathrm{P}=0.586)$ and 5-10 $\mathrm{mm}$ PTMCs $\left(\chi^{2}=5.674, \mathrm{P}=0.059\right)$. However, CEUS was more accurate than 2D ultrasound in differentiating between $2-5 \mathrm{~mm}\left(\chi^{2}=23.380, \mathrm{P}=0.001\right)$ and $5-10 \mathrm{~mm}\left(\chi^{2}=32.734, \mathrm{P}=0.001\right)$ PTMCs. Furthermore, there were significant differences between CEUS and 2D ultrasound used in conjunction with colour Doppler in differentiating between $2-5 \mathrm{~mm}\left(\chi^{2}=23.380, \mathrm{P}=0.001\right)$ and $5-10 \mathrm{~mm}$ 
A

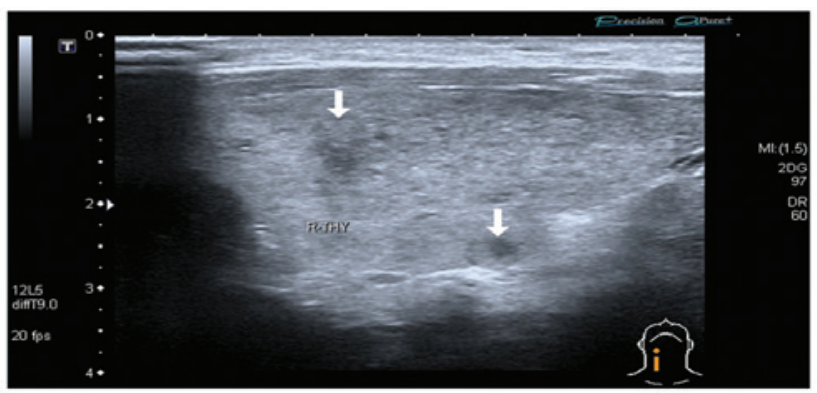

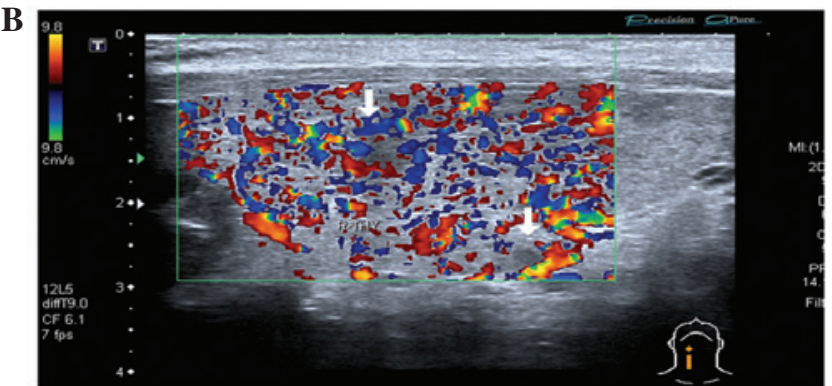

C

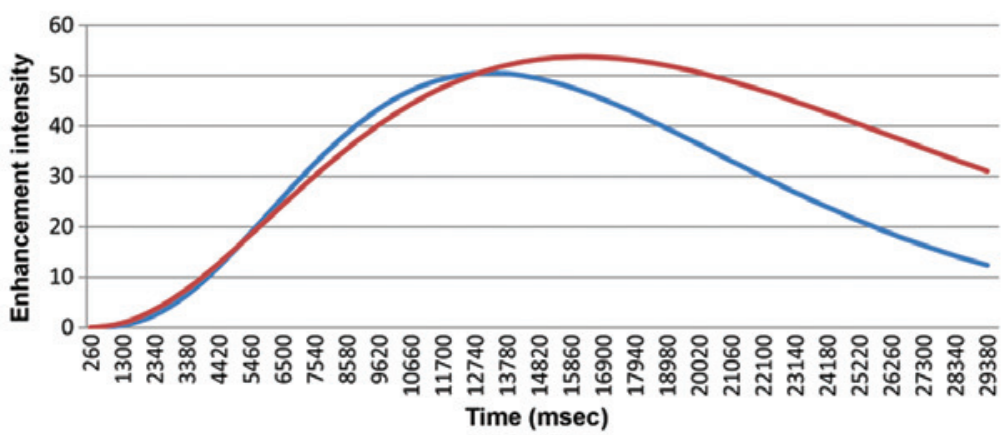

D

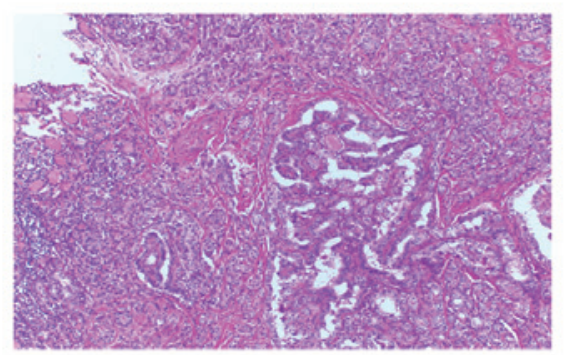

Figure 3. Sonograms of papillary thyroid microcarcinoma with (A) heterogeneous echogenicity and an irregular hypoechoic nodule with an indistinct margin (B-mode ultrasound), (B) rich blood flow in the parenchyma and regular blood flow around the nodule (color Doppler ultrasound). (C) The hypoechoic nodule had a similar arrival time of enhancement as the surrounding normal thyroid tissue. The blue curve represents the thyroid nodule and the red curve the surrounding normal thyroid tissue. Compared with the surrounding normal thyroid tissue, the slope of the descending branch was higher for the thyroid nodule, whereas that of the ascending branch was similar. (D) Hematoxylin and eosin staining of papillary carcinoma with fiber septa and multiple thick-walled vascular proliferation in the interstitial. White arrows indicate the lesion and green lines the CDFI frame.

$\left(\chi^{2}=32.734, \mathrm{P}=0.001\right)$ PTMCs. Finally, CEUS, 2D ultrasound and color Doppler showed no significant difference in their ability to diagnose PTMC. 2D ultrasound used in conjunction with CEUS exhibited the best diagnosis effect. Therefore, CEUS was able to elevate the diagnostic accuracy rate.

\section{Discussion}

In certain patients with PTMC the disease develops slowly, resulting in longer survival rates (7). PTMC with eosinophilic and high cell variants tend to have a higher degree of malignancy and can easily invade tissue outside of the thyroid; for example, the rate of cervical lymph node metastasis is $>80 \%$, resulting in a poor prognosis (8). In the majority of cases, there are no symptoms, however certain lymph node and distant metastases can lead to mortality (7). The early clinical diagnosis of PTMC has therefore become a topic of interest.

The diagnosis rate using palpation is low and the diagnostic specificity of computed tomography/magnetic resonance imaging (CT/MRI) is poor when PTMC nodules are $<10 \mathrm{~mm}$ in diameter (9). Despite this, it has been observed that with an improvement in resolution and examiner's experience, ultrasound can detect micronodules and improve the accuracy of the differential diagnosis of benign and malignant nodules (10). Ultrasound has, therefore, become the preferred imaging diagnostic method for PTMC since it is noninvasive, easy to perform and can have an improved ability in diagnosing PTMC compared with CT/MRI.

Conventional high-frequency ultrasound may be used to diagnose malignant thyroid lesions according to the microcalcification in PTMC (sensitivity, 31.5\%; specificity, 99.1\%) (11). Compared with larger thyroid carcinomas, there is an evident reduction of microcalcification in PTMCs, while color Doppler flow imaging is unable to detect blood flow in the nodules due to their small size (12). Quantitative CEUS is a new technology whose application in the diagnosis of abdominal diseases is developed gradually (13). For example, the use of CEUS in superficial organs is currently being explored; however, its ability to identify PTMC nodules has not yet been widely investigated (14).

Tumor angiogenesis imaging has become increasingly popular. Power Doppler ultrasound (more sensitive than color 
A
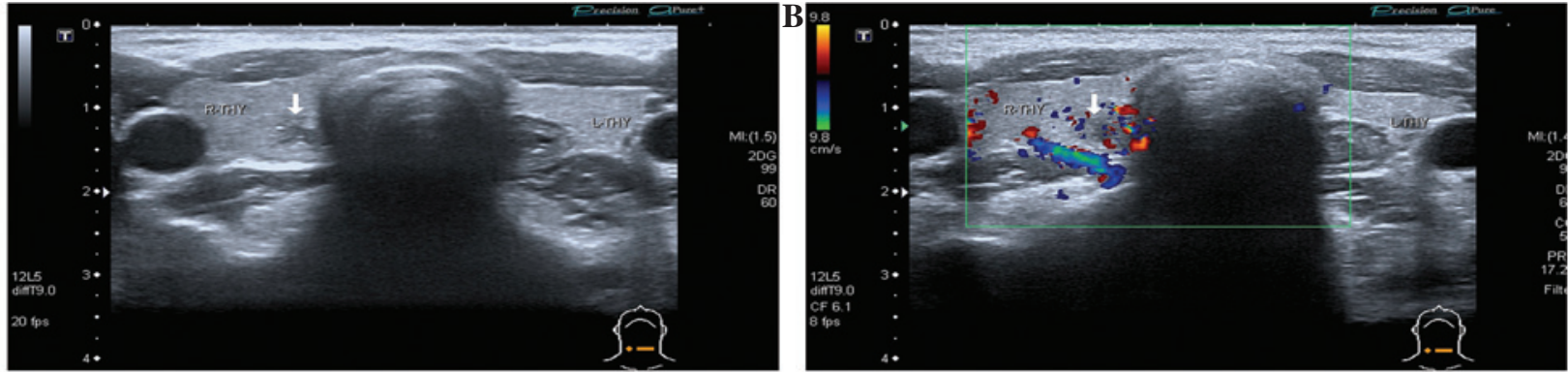

C

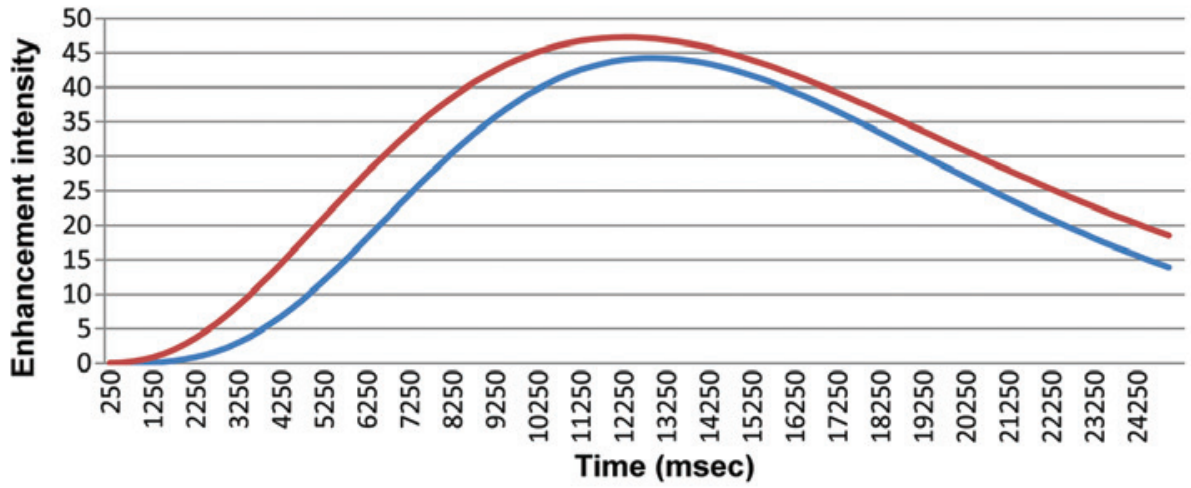

D

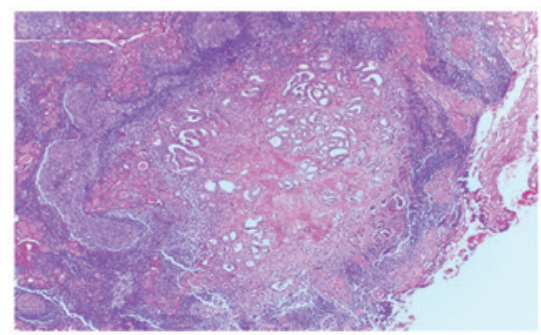

Figure 4. Sonograms from 2D ultrasound of papillary thyroid microcarcinoma with (A) an irregular ill-defined nodule with microcalcification inside (B-mode ultrasound), (B) slightly rich blood flow at the periphery with limited blood flow in the center (color Doppler ultrasound). (C) The hypoechoic nodule had a later arrival time and earlier wash-out time of the enhancement compared with the surrounding normal thyroid tissue. The blue curve represents the thyroid nodule and the red curve the surrounding normal thyroid tissue. Compared with the surrounding normal thyroid tissue, the slope of the ascending branch of the thyroid nodule was lower, whereas that of the descending branch was higher. (D) Hematoxylin and eosin staining of papillary carcinoma with interstitial sclerosis and microvascular proliferation. White arrows indicate the lesion and green lines the CDFI frame.

Doppler) can detect blood flow in arterioles and venules (diameter, $\sim 1 \mathrm{~mm}$; flow velocity, $>10 \mathrm{~mm} / \mathrm{s}$ ); however, it can not detect new blood vessels at the microvessel level. Quantitative CEUS, however, overcomes this limitation and can detect microvessel blood flow velocity at $0.1-10 \mathrm{~mm} / \mathrm{s}$ and observe microvessel perfusion in real-time at high frequency ultrasound, entering CEUS into the speciality of microvessel perfusion imaging (15).

In PTMC, the connective tissue between the epithelium of new blood vessels is not secure, and as a result cancerous vascular thyroid walls are thinner than the walls of a normal thyroid. This results in an incomplete basement membrane and a number of irregular blood vessels with varied thicknesses. This may be why the enhancement of PTCM following contrast medium administration was clearly different from that of normal thyroid parenchyma (16).

The present study observed that 127 nodules demonstrated early or simultaneous echo enhancement compared with the normal thyroid parenchyma, 125 presented inhomogeneous enhancement in dots or strips with an enhanced intensity lower than that of normal thyroid parenchymas, and 128 were inhomogeneously enhanced. In 17 nodules with a rich blood supply, the peak intensity was the same or slightly higher compared with that in the surrounding normal thyroid parenchyma. In addition, 3 nodules exhibited the same edge and periphery enhancement as in thyroid parenchyma, however the center of the nodules showed lower enhancement, and earlier time-to-peak and wash-out time. The pathology of these 3 nodules was concluded to be nodular goiter with papillary thyroid microcarcinoma, as the cancer tissue lay in the centre not in the periphery of the nodules. One nodule demonstrated the same enhancement intensity and arrival time as the surrounding normal thyroid parenchymas; however, this patient was diagnosed with PTMC and Hashimoto's thyroiditis. All cases presented in the current study demonstrate that contrast agents fade earlier in PTMC nodules than in normal thyroid tissue. Therefore, it is hypothesized that the enhancement characteristics of PTCM in CEUS include earlier or simultaneous enhancement, inhomogeneous and low-intensity internal enhancement and earlier wash-out time in comparison to the surrounding normal thyroid parenchyma.

The peak enhanced intensity inside the nodules of certain PTMC were found to be equal to or higher than that of the 
Table IV. Difference among two-dimensional ultrasound, color Doppler and contrast-enhanced ultrasound for diagnosing papillary thyroid microcarcinoma.

\begin{tabular}{|c|c|c|c|c|c|}
\hline Imaging type and nodule diameter & Malignant & Unclear & Benign & $\chi^{2}$-test & P-value \\
\hline \multicolumn{6}{|l|}{ Two-dimensional ultrasound } \\
\hline $2-5 \mathrm{~mm}$ & 31 & 13 & 10 & 1.259 & 0.533 \\
\hline $5-10 \mathrm{~mm}$ & 45 & 22 & 9 & 1.259 & 0.533 \\
\hline \multicolumn{6}{|c|}{ Two-dimensional ultrasound + color Doppler } \\
\hline $2-5 \mathrm{~mm}$ & 36 & 11 & 7 & 5.307 & 0.070 \\
\hline $5-10 \mathrm{~mm}$ & 55 & 19 & 2 & 5.307 & 0.070 \\
\hline \multicolumn{6}{|l|}{ Contrast-enhanced ultrasound } \\
\hline $2-5 \mathrm{~mm}$ & 52 & 2 & 0 & 0.122 & 0.727 \\
\hline $5-10 \mathrm{~mm}$ & 72 & 2 & 0 & 0.122 & 0.727 \\
\hline
\end{tabular}

peripheral parenchyma. In several PTMC nodules, a lack of blood supply was observed as only dot-like or sporadic-like enhancement after SonoVue was administered; however, the evidence of enhancement and rapid wash-out time during the CEUS process was not easily apparent. This may be attributed to the small size of the nodules (diameter, $\leq 5 \mathrm{~mm}$ ), which fail to form the tumor vascular beds and arteriovenous fistulas required to provide sufficient blood supply to the papillary thyroid carcinoma (17). However, the recording of enhancement may be influenced by the condition of the apparatus and the experience of the operator, as evidence of enhancement can be missed and be recorded as having no change in the nodule enhancement and wash-out times. Under a repeated and careful observation and analysis, an earlier wash-out time may be observed in PTMC nodules in comparison to normal thyroid parenchyma (18).

Color Doppler and 2D ultrasound may potentially form the foundation of PTMC diagnosis $(19,20)$. However, as the majority of PTCM nodules have a reduced blood supply, CEUS is helpful in detecting and displaying microvessels, detecting low-speed blood flow and improving the sensitivity of diagnosis $(21,22)$. Therefore, CEUS may be a novel and valuable diagnostic tool for the identification and diagnosis of PTCM.

In conclusion, PTMC enhancement washed out faster than in normal thyroid parenchyma. The PTMC characteristics of CEUS may improve the accuracy of diagnosis and provide valuable information for the treatment of the disease.

\section{Acknowledgements}

The present study was financially supported by grants from the SJTU Medicine Engineering Interdisciplinary Research Fund (no. YG2013MS53), the Shanghai Municipal Natural Science Foundation (no. 12ZR1422600), the National Nature Science Foundation of China (no. 81371574) and the Research Fund for the Doctoral Program of Higher Education of Chinam (no. 20120073120100).

\section{References}

1. Colonna M, Uhry Z, Guizard AV, Delafosse P, Schvartz C, Belot A, Grosclaude P and FRANCIM network: Recent trends in incidence, geographical distribution, and survival of papillary thyroid cancer in France. Cancer Epidemiol 39: 511-518, 2015.
2. Roti E, degli Uberti EC, Bondanelli M and Braverman LE: Thyroid papillary microcarcinoma: A descriptive and meta-analysis study. Eur J Endocrinol 159: 659-673,2008.

3. Hughes DT, Haymart MR, Miller BS, Gauger PG and Doherty GM: The most commonly occurring papillary thyroid cancer in the United States is now a microcarcinoma in a patient older than 45 years. Thyroid 21: 231-236, 2011.

4. Ito Y, Tomoda C, Uruno T, Takamura Y, Miya A, Kobayashi K, Matsuzuka F, Kuma K and Miyauchi A: Papillary microcarcinoma of the thyroid: How should it be treated? World J Surg 28: 1115-1121, 2004.

5. Pelizzo MR, Boschin IM, Toniato A, Piotto A, Bernante P, Pagetta C, Rampin L and Rubello D: Papillary thyroid microcarcinoma (PTMC): Prognostic factors, management and outcome in 403 patients. Eur J Surg Oncol 32: 1144-1148, 2006.

6. Smith-Bindman R, Lebda P, Feldstein VA, Sellami D, Goldstein RB, Brasic N, Jin C and Kornak J: Risk of thyroid cancer based on thyroid ultrasound imaging characteristicscs: Results of a population-based study. JAMA Intern Med 173: 1788-1796, 2013.

7. Pelizzo MR, Boschin IM, Toniato A, Pagetta C, Piotto A, Bernante P, Casara D, Pennelli G and Rubello D: Natural history, diagnosis, treatment and outcome of papillary thyroid microcarcinoma (PTMC): A mono-institutional 12-year experience. Nucl Med Commun 25: 547-552, 2004.

8. Pelizzo MR, Boschin IM, Toniato A, Pagetta C, Piotto A Bernante P, Casara D, Pennelli G and Rubello D: Natural history, diagnosis, treatment and outcome of papillary thyroid microcarcinoma (PTMC): A mono-institutional 12-year experience. Nucl Med Commun 25: 547-552, 2004.

9. Cosgrove D: Microbubble enhancement of tumour neovascularity. Eur Radiol 9 (Suppl 3): S413-S414, 1999.

10. Mitchell JC, Parangi S: Angiogenesis in benign and malignant thyroid disease. Thyroid 15: 494-510, 2005.

11. Nemec U, Nemec SF, Novotny C, Weber M, Czerny C and Krestan CR: Quantitative evaluation of contrast-enhanced ultrasound after intravenous administration of a microbubble contrast agent for differentiation of benign and malignant thyroid nodules: Assessment of diagnostic accuracy. Eur Radiol 22: 1357-1365, 2012.

12. Agha A, Jung EM, Janke M, Hornung M, Georgieva M, Schlitt HJ, Schreyer AG, Strosczcynski C and Schleder S: Preoperative diagnosis of thyroid adenomas using high resolution contrast-enhanced ultrasound (CEUS). Clin Hemorheol Microcirc 55: 403-409, 2013.

13. Seo YL, Yoon DY, Baek S, Ku YJ, Rho YS, Chung EJ and Koh SH: Detection of neck recurrence in patients with differentiated thyroid cancer: comparison of ultrasound, contrast-enhanced CT and (18)F-FDG PET/CT using surgical pathology as a reference standard: (ultrasound vs. CT vs. (18) F-FDG PET/CT in recurrent thyroid cancer). Eur Radiol 22: 2246-2254, 2012.

14. Molinari F, Mantovani A, Deandrea M, Limone P, Garberoglio R and Suri JS: Characterization of single thyroid nodules by contrast-enhanced 3-D ultrasound. Ultrasound Med Biol 36:1616-1625, 2010. 
15. Deng J,Zhou P, Tian SM,Zhang L, Li JL and Qian Y: Comparison of diagnostic efficacy of contrast-enhanced ultrasound, acoustic radiation force impulse imaging, and their combined use in differentiating focal solid thyroid nodules. PLoS One 9: e90674, 2014.

16. Acharya UR, Vinitha Sree S, Krishnan MM, Molinari F, Garberoglio R and Suri JS: Non-invasive automated 3D thyroid lesion classification in ultrasound: A class of ThyroScan ${ }^{\mathrm{TM}}$ systems. Ultrasonics 52: 508-520, 2012.

17. Hornung M, Jung EM, Georgieva M, Schlitt HJ, Stroszczynski C and Agha A: Detection of microvascularization of thyroid carcinomas using linear high resolution contrast-enhanced ultrasonography (CEUS). Clin Hemorheol Microcirc 52: 197-203, 2012.

18. Cantisani V, Consorti F, Guerrisi A, Guerrisi I, Ricci P, Di Segni M, Mancuso E, Scardella L, Milazzo F, D’Ambrosio F and Antonaci A: Prospective comparative evaluation of quantitative-elastosonography (Q-elastography) and contrast-enhanced ultrasound for the evaluation of thyroid nodules: Preliminary experience. Eur J Radio 182: 1892-1898, 2013.
19. Giusti M, Orlandi D, Melle G, Massa B, Silvestri E, Minuto F and Turtulici G: Is there a real diagnostic impact of elastosonography and contrast-enhanced ultrasonography in the management of thyroid nodules? J Zhejiang Univ Sci B 14: 195-206, 2013

20. Agha A, Hornung M, Rennert J, Uller W, Lighvani H, Schlitt HJ and Jung EM: Contrast-enhanced ultrasonography for localization of pathologic glands in patients with primary hyperparathyroidism. Surgery 151: 580-586, 2012.

21. Dietrich CF, Ignee A, Hocke M, Schreiber-Dietrich D and Greis C: Pitfalls and artefacts using contrast enhanced ultrasound. Z Gastroenterol 49: 350-356, 2011.

22. Friedrich-Rust M, Sperber A, Holzer K, Diener J, Grünwald F, Badenhoop K, Weber S, Kriener S, Herrmann E, Bechstein WO, Zeuzem S and Bojunga J: Real-time elastography and contrast-enhanced ultrasound for the assessment of thyroid nodules. Exp Clin Endocrinol Diabetes 118: 602-609, 2010. 